PROCEEDINGS OF THE

AMERICAN MATHEMATICAL SOCIETY

Volume 125, Number 3, March 1997, Pages 679-684

S 0002-9939(97)03658-7

\title{
SIMPLE CONNECTEDNESS OF PROJECTIVE VARIETIES
}

\author{
STEVEN DALE CUTKOSKY
}

(Communicated by Wolmer V. Vasconcelos)

\begin{abstract}
A Lefschetz type theorem is proven relating the algebraic fundamental group of a smooth projective variety $X$ to the algebraic fundamental group of a subvariety set theoretically defined by $\leq \operatorname{dim}(X)-2$ forms.
\end{abstract}

In this paper we prove a generalization of Grothendieck's Lefschetz theorem for complete intersections (SGA2 XII 3.5). Our result is:

Theorem 1. Suppose that $k$ is a field, $W$ is a smooth, geometrically connected subvariety of $\mathbf{P}_{k}^{m}$ of dimension $n$ and $Z \subset W$ is a closed subscheme set theoretically defined by the vanishing of $r$ forms of $\mathbf{P}_{k}^{m}$ on $W$.

1. If $r \leq n-1$ then $Z$ is geometrically connected and there is a surjection $\pi_{1}(Z) \rightarrow \pi_{1}(W)$.

2. If $r \leq n-2$, then $\pi_{1}(Z) \cong \pi_{1}(W)$.

Corollary 2. Suppose that $k$ is a field and $Z \subset \mathbf{P}_{k}^{n}$ is a closed subscheme set theoretically defined by $r$ forms.

1. If $r \leq n-1$ then $Z$ is geometrically connected.

2. If $r \leq n-2$, then $\pi_{1}(Z) \cong \operatorname{Gal}(\bar{k} / k)$ where $\bar{k}$ is an algebraic closure of $k$.

The corresponding theorem for the topological fundamental group of a complex projective variety follows from Hamm [H] and the Theorem of II 1.2 in [GM]. Their proofs use different methods (Morse theory) and do not extend to positive characteristic.

$\pi_{1}(X)$ will denote the algebraic fundamental group of a scheme $X$. If $k$ is a field, $\bar{k}$ will denote an algebraic closure of $k$.

\section{SiMPLE CONNECTEDNESS}

Proof of the first half of 1 . of Theorem 1. Suppose that $r \leq n-1$. Let $K$ be an extension field of $k$. Let $(A, m)$ be the local ring of the homogeneous coordinate ring of $W \otimes_{k} K, \hat{A}$ be completion at $m$. Suppose that $Z$ is set theoretically defined by $r$ forms $f_{1}, \ldots, f_{r} \in A . \quad r \leq(n+1)-2 \operatorname{implies} \operatorname{spec}\left(\hat{A} /\left(f_{1}, \ldots, f_{r}\right)\right)-\hat{m}$ is connected by Corollary 4 to Theorem $1[\mathrm{~F}]$, since $\hat{A}$ is a domain. Hence $Z$ is geometrically connected.

Definition 3. A morphism $f: X \rightarrow Y$ is separable if $f$ is flat and for any $y \in Y$, $X \times_{Y} k(y)$ is geometrically reduced over $k(y)$.

Received by the editors September 14, 1995.

1991 Mathematics Subject Classification. Primary 14F35, 14E20.

Partially supported by NSF.

(C)1997 American Mathematical Society 
Lemma 4. Suppose that $Y$ is a noetherian scheme, $f: X \rightarrow Y$ is a finite type morphism. Let

$$
A=\left\{y \in Y \mid X_{y} \text { is geometrically reduced over } k(y)\right\} .
$$

Then

1. A is a constructible subset of $Y$.

2. If $g: Y^{\prime} \rightarrow Y$ is a morphism then

$$
g^{-1}(A)=\left\{y^{\prime} \in Y^{\prime} \mid X \times_{Y} Y_{y^{\prime}}^{\prime} \text { is geometrically reduced over } k\left(y^{\prime}\right)\right\} .
$$

Proof. This is EGA IV.9.7.7 and IV.9.2.2 (iv).

Lemma 5. Suppose that $Y$ is a noetherian scheme and $Z \subset Y$ is a constructible subset. Let $\bar{Z}$ be the closure of $Z$ in $Y$. If $Z$ contains no generic points of codimension one irreducible subschemes of $Y$, then $\bar{Z}$ has codimension $\geq 2$ in $Y$.

Proof. This follows from the fact proved in EGA $0_{I I I} 9.2 .3$ that a constructible subset of an irreducible subset $W$ of $Y$ is dense in $W$ if and only if it contains a nonempty open subset.

We need to generalize to morphisms which are not separable the exact homotopy sequence for proper separable morphisms of SGA1 X 1.4 and Theorem 6.3.2.1 [M].

Theorem 6. Suppose that $Y$ is a connected regular scheme, $X$ is normal, $f: X \rightarrow$ $Y$ is a proper morphism such that $f$ is separable in codimension one (in $Y$ ) and $f_{*} \mathcal{O}_{X} \cong \mathcal{O}_{Y}$. Let $y \in Y$ be the generic point, $\overline{X_{y}}=X \times_{Y} \overline{k(y)}$. Then there is a natural right exact sequence

$$
\pi_{1}\left(\overline{X_{y}}\right) \stackrel{\Phi}{\rightarrow} \pi_{1}(X) \stackrel{\Psi}{\rightarrow} \pi_{1}(Y) \rightarrow 0 .
$$

Proof. The proofs that $\Psi$ is surjective and $\Psi \circ \Phi=0$ are exactly as in the proof of Theorem 6.3.2.1 $[\mathrm{M}]$. We must prove that $\operatorname{Image}(\Phi) \supset \operatorname{kernel}(\Psi)$. By the criterion of 5.2.4 [M], we must show that if $g: X^{\prime} \rightarrow X$ is a connected étale cover of $X$ and the base change $\bar{g}: X^{\prime} \times_{X} \overline{X_{y}} \rightarrow \overline{X_{y}}$ has a section $\sigma$ over $\overline{X_{y}}$, then there exists a connected étale cover $Y^{\prime} / Y$ such that $X^{\prime} \cong X \times_{Y} Y^{\prime}$.

Suppose that $g: X^{\prime} \rightarrow X$ is such a morphism. $f \circ g$ is proper and separable in codimension one by Lemma 6.3.2.2 [M]. Let $X^{\prime} \stackrel{h^{\prime}}{\rightarrow} Y^{\prime} \rightarrow Y$ be the stein factorization of $f \circ g$. By Theorem 6.2.1 [M] $Y^{\prime} \rightarrow Y$ is étale in codimension one. $Y^{\prime}$ is normal since $X^{\prime}$ is. By purity of branch locus for regular schemes (c.f. SGA1 X 3.1) $Y^{\prime} \rightarrow Y$ is étale.

It remains to show that the natural map $\alpha: X^{\prime} \rightarrow X \times_{Y} Y^{\prime}$ is an isomorphism. This is shown exactly as on pages 115-116 in the proof of Theorem 6.3.2.1 [M].

Corollary 7. Suppose that $Y$ is a connected regular excellent scheme, $X$ is normal, $f: X \rightarrow Y$ is a proper morphism such that $f$ is separable in codimension 1, and $f_{*} \mathcal{O}_{X}=\mathcal{O}_{Y}$. Let $z_{0} \in Y$ be a point and $z_{1} \in Y$ be the generic point. Let $\bar{X}_{0}=X \times_{Y} \overline{k\left(z_{0}\right)}, \bar{X}_{1}=X \times_{Y} \overline{k\left(z_{1}\right)}$. Then there is a natural surjection

$$
\pi_{1}\left(\overline{X_{1}}\right) \rightarrow \pi_{1}\left(\overline{X_{0}}\right) .
$$

Proof. Let $A=\mathcal{O}_{Y}, z_{0}, \hat{A}$ be the completion of $A$ at its maximal ideal, $Y^{\prime}=$ $\operatorname{spec}(\hat{A})$. Let $z_{1}^{\prime}$ be the generic point and $z_{0}^{\prime}$ be the closed point of $Y^{\prime}, X^{\prime}=X \times_{Y} Y^{\prime}$, with natural morphism $f^{\prime}: X^{\prime} \rightarrow Y^{\prime}$. $f^{\prime}$ is proper and $f_{*}^{\prime} \mathcal{O}_{X^{\prime}}=\mathcal{O}_{Y^{\prime}}$. $X^{\prime}$ is normal since $X$ is excellent. If $P \subset \hat{A}$ is a height one prime then $\operatorname{ht}(P \cap A) \leq 1$. By Lemma 
$4 f^{\prime}$ is separable in codimension 1. Let $\overline{X_{0}^{\prime}}=X^{\prime} \times_{Y^{\prime}} \overline{k\left(z_{0}^{\prime}\right)}, \overline{X_{1}^{\prime}}=X^{\prime} \times_{Y^{\prime}}, \overline{k\left(z_{1}^{\prime}\right)}$. $\pi_{1}\left(\overline{X_{0}^{\prime}}\right)=\pi_{1}\left(\overline{X_{0}}\right)$ and $\pi_{1}\left(\overline{X_{1}^{\prime}}\right) \cong \pi_{1}\left(\overline{X_{1}}\right)$ by Proposition 7.3.2 [M]. By careful choice of basepoints we have a commutative diagram:

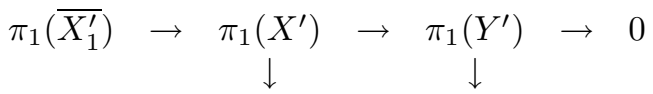

$$
\begin{aligned}
& 0 \rightarrow \pi_{1}\left(\overline{X_{0}^{\prime}}\right) \rightarrow \pi_{1}\left(X^{\prime}\right) \rightarrow \pi_{1}\left(Y^{\prime}\right) \rightarrow 0
\end{aligned}
$$

The top row is right exact by Theorem 6 . The bottom row is exact by SGA1 $\mathrm{X}$ 2.2 or Theorem 8.1.1 $[\mathrm{M}]$. The two vertical maps are the identity. Hence there is a surjection $\pi_{1}\left(\overline{X_{1}}\right) \rightarrow \pi_{1}\left(\overline{X_{0}}\right)$.

Let notation be as in the statement of Theorem 1 . Let $I_{W} \subset k\left[x_{0}, \ldots x_{m}\right]$ be the homogeneous ideal of $W$. Suppose that $r \leq n-1$ and $k$ is algebraically closed. There exist integers $d_{1}$ and $d_{2}$, where we can assume that

$$
H^{1}\left(\mathbf{P}_{k}^{m}, \mathcal{O}\left(d_{1}\right) \otimes I_{W}\right)=H^{1}\left(\mathbf{P}_{k}^{m}, \mathcal{O}\left(d_{2}\right) \otimes I_{W}\right)=0
$$

and we can take $d_{2}$ arbitrarily large relative to $d_{1}$ such that $Z$ is defined set theoretically by the vanishing of $r-1$ forms $f_{1}, \ldots, f_{r-1}$ of degree $d_{1}$ and a form $f_{r}$ of degree $d_{2}$. Let $t_{1}=h^{0}\left(W, \mathcal{O}_{W}\left(d_{1}\right)\right), t_{2}=h^{0}\left(W, \mathcal{O}_{W}\left(d_{2}\right)\right)$. Let $a_{1}^{I}, \ldots, a_{r-1}^{I}, b_{r}^{J}$ be $(r-1) t_{1}+t_{2}$ indeterminates, where $I$ indexes a basis $\sigma_{1}, \ldots, \sigma_{t_{1}}$ of $H^{0}\left(W, \mathcal{O}_{W}\left(d_{1}\right)\right)$ and $J$ indexes a basis $\tau_{1}, \ldots, \tau_{t_{2}}$ of $H^{0}\left(W, \mathcal{O}_{W}\left(d_{2}\right)\right)$. Let

$$
\begin{gathered}
F_{1}=\sum_{I=1}^{t_{1}} a_{1}^{I} \sigma_{I}, \ldots, F_{r-1}=\sum_{I=1}^{t_{1}} a_{r-1}^{I} \sigma_{I}, F_{r}=\sum_{J=1}^{t_{2}} b_{r}^{J} \tau_{J} . \\
F_{1}, \ldots, F_{r} \in\left(k\left[x_{0}, \ldots, x_{m}\right] / I_{W}\right)\left[a_{1}^{I}, \ldots, a_{r-1}^{I}, b_{r}^{J}\right] .
\end{gathered}
$$

Let $Y=\operatorname{Proj}\left(k\left[a_{1}^{I}, \ldots, a_{r-1}^{I}, b_{r}^{J}\right]\right)$,

$$
X=V\left(F_{1}, \ldots, F_{r}\right) \subset Y \times W
$$

be the subscheme determined by $F_{1}, \ldots, F_{r}$. There is a natural projective morphism $f: X \rightarrow Y$. Let $p \in Y$ be the closed point such that $\left(X_{p}\right)_{\text {red }} \cong Z_{\text {red }}$.

Proposition 8. Let notation be as in the above paragraph.

1. $X$ is smooth over $k$.

2. $f_{*} \mathcal{O}_{X}=\mathcal{O}_{Y}$.

3. Let $E=\left\{y \in Y \mid X_{y}\right.$ is not geometrically reduced over $\left.k(y)\right\}, \bar{E}$ be the closure of $E$ in $Y$. Then $\operatorname{codim}_{Y}(\bar{E}) \geq 2$.

4. Let $F=\left\{y \in Y \mid\right.$ there exists $x \in f^{-1}(y)$ such that $\mathcal{O}_{X, x}$ is not flat over $\left.\mathcal{O}_{Y, y}\right\}$. Then $\operatorname{codim}_{Y}(F) \geq 2$.

Note That $E$ is constructible and $F$ is closed.

Proof. $X$ is smooth over $k$ by the Jacobian criterion.

Let $X \rightarrow Y^{\prime} \rightarrow Y$ be the Stein factorization of $f . Y^{\prime} \rightarrow Y$ is dominant, finite and $Y^{\prime}$ is normal. By Bertini's theorem (cf. II 8.18 [Ha]), there exists a dense open subset $U$ of $Y$ such that if $q \in Y$ is a closed point, then $X_{q} \subset W$ is a smooth irreducible complete intersection of dimension $\geq 1$. Hence $Y^{\prime} \rightarrow Y$ is generically 1-1. Thus $Y^{\prime}=Y$ by Zariski's Main Theorem, and $f_{*} \mathcal{O}_{X}=\mathcal{O}_{Y}$.

Given $\overline{a_{i}^{I}} \in k$ (with at least one $\overline{a_{i}^{I}} \neq 0$ ), let

$$
V\left(\overline{a_{i}^{I}}\right)=\operatorname{Proj}\left(k\left[a_{1}^{I}, \ldots, a_{(r-1)}^{I}, b_{r}^{J}\right] /\left(\overline{a_{i}^{I}} a_{k}^{K}-\overline{a_{k}^{K}} a_{i}^{I}\right)\right) \subset Y .
$$


Each $V\left(\overline{a_{i}^{I}}\right) \cong \mathbf{P}^{t_{2}}$. The union of $V\left(\overline{a_{i}^{I}}\right)$ over all choices of $\overline{a_{i}^{I}}$ is $Y$. Let

$$
D=\operatorname{Proj}\left(k\left[a_{1}^{I}, \ldots, a_{(r-1)}^{I}, b_{r}^{J}\right] /\left(a_{1}^{I}, \ldots, a_{(r-1)}^{I}\right)\right) \cong \operatorname{Proj}\left(k\left[b_{r}^{J}\right]\right) .
$$

Let $\bar{F}_{1}, \ldots, \bar{F}_{r-1} \in k\left[x_{0}, \ldots, x_{m}\right] / I_{W}$ be the corresponding specializations of $F_{1}, \ldots, F_{r-1}$ over $\left(a_{i}^{I}\right) \rightarrow\left(\overline{a_{i}^{I}}\right)$.

We can choose $\overline{a_{i}^{I}}$ such that if $\Gamma$ is a codimension one integral component of $F \cup \bar{E}$ then $\Gamma \cap V\left(\overline{a_{i}^{I}}\right)$ is not contained in $D$ and if $T$ is the subscheme of $W$ determined by the vanishing of $\bar{F}_{1}, \ldots, \bar{F}_{r-1}$, then $T$ is a smooth subvariety of $W$ of dimension $n-(r-1) \geq 2$.

Let $V=V\left(\overline{a_{i}^{I}}\right)-D . V$ parametrizes the intersections of $T$ with the zero locus of sections of $H^{0}\left(W, \mathcal{O}_{W}\left(d_{2}\right)\right)$. Hence we have a natural identification

$$
V=\mathbf{V}\left(S\left(H^{0}\left(W, \mathcal{O}_{W}\left(d_{2}\right)\right)^{*}\right)\right)
$$

where $*$ denotes dual $k$ vector space.

To show that $\operatorname{codim}_{Y}(\bar{E}) \geq 2$ and $\operatorname{codim}_{Y}(F) \geq 2$, it suffices by our construction of $V$ to show that $\operatorname{codim}_{V}(V \cap \bar{E}) \geq 2$ and $\operatorname{codim}_{V}(V \cap F) \geq 2$.

Let $I_{T}=\left(\bar{F}_{1}, \ldots, \bar{F}_{r-1}\right)+I_{W} \subset k\left[x_{0}, \ldots, x_{m}\right] . I_{T}$ is the homogeneous ideal of $T$. Recall that for fixed $T$, we are free to choose $d_{2}$ arbitrarily large. Since $\mathcal{O}(1)$ is ample, $\operatorname{dim} T \geq 2$, we can choose $d_{2}$ so that

i) $H^{1}\left(W, \mathcal{O}_{W}\left(d_{2}\right) \otimes I_{T}\right)=0$.

ii) $h^{0}\left(W, \mathcal{O}_{W}\left(d_{2}\right) \otimes I_{T}\right)<h^{0}\left(W, \mathcal{O}_{W}\left(d_{2}\right)\right)-1$.

iii) If $D, C$ are nonzero effective divisors on $T$ such that $\mathcal{O}_{T}(D+C) \cong \mathcal{O}_{T}\left(d_{2}\right)$, then

$$
h^{0}\left(T, \mathcal{O}_{T}(C)\right)<h^{0}\left(T, \mathcal{O}_{T}\left(d_{2}\right)\right)-\operatorname{dim} \operatorname{Pic}(T)-1 .
$$

Assertions i) and ii) follow from Serre's vanishing theorem, and since $h^{0}\left(W, \mathcal{O}_{W}(d)\right)$ is a polynomial in $d$ of degree $n$ for large $d$ and $h^{0}\left(T, \mathcal{O}_{T}(d)\right)$ is a polynomial in $d$ of degree $n-(r-1) \geq 2$ for large $d$.

Now we will verify iii). Let $s=\operatorname{dim} \operatorname{Pic}(T)+2$. If $d_{2}$ is sufficiently large, $\mathcal{O}_{T}\left(d_{2}\right)$ has the property that if $p_{1}, \ldots, p_{s}$ are any distinct closed points in $T$, then

$$
h^{0}\left(T, \mathcal{O}_{T}\left(d_{2}\right) \otimes \mathcal{O}_{T}\left(-p_{1}-\ldots-p_{s}\right)\right)=h^{0}\left(T, \mathcal{O}_{T}\left(d_{2}\right)\right)-s .
$$

If $D, C$ are as in iii), and $p_{1}, \ldots, p_{s}$ are distinct closed points of $D$, then

$$
\begin{aligned}
h^{0}\left(T, \mathcal{O}_{T}(C)\right) & \left.=h^{0}\left(T, \mathcal{O}_{T}\left(d_{2}\right)\right) \otimes \mathcal{O}_{T}(-D)\right) \\
& \leq h^{0}\left(T, \mathcal{O}_{T}\left(d_{2}\right) \otimes \mathcal{O}_{T}\left(-p_{1}-\ldots-p_{s}\right)\right) \\
& <h^{0}\left(T, \mathcal{O}_{T}\left(d_{2}\right)\right)-\operatorname{dim} \operatorname{Pic}(T)-1
\end{aligned}
$$

and iii) holds.

By i) we have a natural exact sequence

$$
0 \rightarrow H^{0}\left(W, \mathcal{O}_{W}\left(d_{2}\right) \otimes I_{T}\right) \rightarrow H^{0}\left(W, \mathcal{O}_{W}\left(d_{2}\right)\right) \rightarrow H^{0}\left(T, \mathcal{O}_{T}\left(d_{2}\right)\right) \rightarrow 0 .
$$

Let $H \rightarrow \mathbf{P}\left(H^{0}\left(T, \mathcal{O}_{T}\left(d_{2}\right)\right)^{*}\right)$ be the universal family parametrizing the subschemes of $T$ given by vanishing of sections of $H^{0}\left(T, \mathcal{O}_{T}\left(d_{2}\right)\right)$. Let

$$
V^{\prime}=\mathbf{V}\left(H^{0}\left(W, \mathcal{O}_{W}\left(d_{2}\right)\right)^{*}\right)-\mathbf{V}\left(H^{0}\left(W, \mathcal{O}_{W}\left(d_{2}\right) \otimes I_{T}\right)^{*}\right) .
$$

That is, $V^{\prime}$ is the complement of $\mathbf{V}\left(H^{0}\left(W, \mathcal{O}_{W}\left(d_{2}\right) \otimes I_{T}\right)^{*}\right)$ in

$$
V=\mathbf{V}\left(H^{0}\left(W, \mathcal{O}_{W}\left(d_{2}\right)\right)^{*}\right) .
$$


(1) gives a natural surjection

$$
\lambda: V^{\prime} \rightarrow \mathbf{P}\left(H^{0}\left(T, \mathcal{O}_{T}\left(d_{2}\right)\right)^{*}\right)
$$

such that $X_{V^{\prime}}=\lambda^{*}(H)$.

We have $V \cap F=\mathbf{V}\left(H^{0}\left(W, \mathcal{O}_{W}\left(d_{2}\right) \otimes I_{T}\right)^{*}\right)$, so that $\operatorname{codim}_{V}(V \cap F) \geq 2$ by ii and assertion 4) follows.

If $\zeta \in V$ is the generic point of a codimension 1 subvariety of $V$, then $\zeta \in V^{\prime}$ by ii), and the closure of $\lambda(\zeta)$ has codimension $\leq 1$ in $\mathbf{P}\left(H^{0}\left(T, \mathcal{O}_{T}\left(d_{2}\right)\right)^{*}\right)$. Let $\alpha=\lambda(\zeta)$. By Lemma $4 X_{\zeta}$ is geometrically reduced over $k(\zeta)$ if and only if $H_{\alpha}$ is geometrically reduced over $k(\alpha)$.

Let $B^{\prime}$ be the closure of $\alpha$ in $\mathbf{P}\left(H^{0}\left(T, \mathcal{O}_{T}\left(d_{2}\right)\right)^{*}\right)$. There is a finite radicial morphism $\tau: B \rightarrow B^{\prime}$ such that if $\beta$ is the generic point of $B,\left(H_{\beta}\right)_{\text {red }}$ is geometrically reduced over $k(\beta)$ (cf. IV.4.6.6 EGA). If $H_{\beta}$ is not reduced, then there exists a dense open $U \subset B$ and a flat map $\left(H_{B}\right)_{\text {red }} \times_{B} U \rightarrow U$ such that the fibers over closed points of $U$ are pairwise distinct subschemes of $T$, each given by the vanishing of a section of $H^{0}\left(T, \mathcal{O}_{T}(C)\right)$ for some effective divisor $C$ on $T$ with $h^{0}\left(T, \mathcal{O}_{T}\left(d_{2}\right) \otimes \mathcal{O}_{T}(-C)\right)>0$, and where each fiber has a common Hilbert polynomial $P$. Let $\operatorname{Hilb}^{P}$ be the component of the Hilbert scheme of $T$ of subschemes with the Hilbert polynomial $P$.

There exists an immersion $U \rightarrow \operatorname{Hilb}^{P}$ such that $\left(H_{B}\right)_{r e d} \times_{B} U$ is the pullback of the universal family over $\operatorname{Hilb}^{P}$.

There is a morphism $\gamma: \operatorname{Hilb}^{P} \rightarrow \operatorname{Pic}(T)$ where the fiber containing the point corresponding to the subscheme $C$ is $\mathbf{P}\left(H^{0}\left(T, \mathcal{O}_{T}(C)\right)^{*}\right)$. By iii),

$$
\operatorname{dimHilb}^{P} \leq \operatorname{dim} \mathbf{P}\left(H^{0}\left(T, \mathcal{O}_{T}\left(d_{2}\right)\right)^{*}\right)-2 .
$$

Hence $\operatorname{dim}\left(B^{\prime}\right)=\operatorname{dim}(U) \leq \operatorname{dim} \mathbf{P}\left(H^{0}\left(T, \mathcal{O}_{T}\left(d_{2}\right)\right)^{*}\right)-2$. This shows that $\lambda(\beta)=\alpha$ has codimension $>1$ in $\mathbf{P}\left(H^{0}\left(T, \mathcal{O}_{T}\left(d_{2}\right)\right)^{*}\right)$, a contradiction, so that $X_{\zeta}$ is geometrically reduced over $k(\zeta) \operatorname{codim}_{V}(V \cap \bar{E}) \geq 2$ so that $\operatorname{codim}_{Y}(\bar{E}) \geq 2$.

Proof of Theorem 1. First suppose that $k$ is algebraically closed. Consider the map $f: X \rightarrow Y$ defined before Proposition 8. By Proposition 8 the assumptions of Corollary 7 are satisfied. Hence there is a surjection $\pi_{1}\left(\overline{X_{1}}\right) \rightarrow \pi_{1}\left(X_{p}\right)=\pi_{1}(Z)$. $X_{1} \subset W \otimes_{k} k\left(a_{i}^{I}, a_{r}^{J}\right)$ is a smooth irreducible complete intersection of dimension $\geq 1$ under the assumptions of 1 ) and of dimension $\geq 2$ under the assumptions of 2). The composite map

$$
\pi_{1}\left(\bar{X}_{1}\right) \rightarrow \pi_{1}(Z) \rightarrow \pi_{1}(W)
$$

is a surjection under the assumptions of 1) and is an isomorphism under the assumptions of 2) by SGA2 XII 3.5 and Proposition 7.3.2 [M]. If $k$ is not algebraically closed the conclusions of 1) and 2) now hold by SGA1 IX 6.1 or Theorem 8.1.1 [M].

\section{REFERENCES}

[Z] O. Zariski, The Theorem of Riemann-Roch for high multiples of an effective divisor on an algebraic surface, Ann. Math. 76 (1962) 560-616. MR 25:5065

[CS] S.D. Cutkosky and H. Srinivasan, Local fundamental groups of surface singularities in characteristic p, Comment. Math. Helvetici, 68 (1993), 319-332. MR 94h:14035

[C] S.D. Cutkosky, Purity of branch locus and Lefschetz theorems, Compositio Math. 96, (1995) 173-195. CMP 95:11

[F] G. Faltings, Algebraisation of some formal vector bundles, Annals of Mathematics, 110 (1979), 501-514. MR 82e:14011 
[GM] M. Goresky and R. MacPherson, Stratified Morse Theory, Springer-Verlag, Berlin (1988) MR 90d:57039

[EGA] A. Grothendieck and J. Dieudonne, EGA I-IV, Publ. Math. IHES 4,8,11,17,24,28,32 MR 29:1210; MR 30:3885; MR 33:7330; MR 36:177a,b,c,178; MR 39:220

[SGA1] A. Grothendieck, Revetements étale et groupe fondemental, Lecture notes in Math 224, Springer-Verlag, Heidelberg (1971) MR 50:7129

[SGA2] _ Cohomologie locale des Faisceaux coherents et Theoremes de Lefschetz locaux et globaux, North-Holland, Amsterdam (1968). MR 57:16294

$[\mathrm{H}] \quad$ H.Hamm, Lefschetz theorems for singular varieties, Singularities, Proc. Symp. Pure Math Vol. 40, Amer. Math. Soc., Providence, RI (1983), pp. 547-557. MR 85d:32025

[Ha] R. Hartshorne,Algebraic Geometry, Springer, New York (1977). MR 57:3116

[L] G. Lyubeznik, Etale cohomological dimension and the topology of algebraic varieties, Annals Math. 137 (1993), 71-128. MR 93m:14016

[M] J. Murre, Lectures on an introduction to Grothendieck's theory of the fundamental group, Lecture notes, Tata Institute of Fundamental Research, Bombay, (1967). MR 46:1794

Department of Mathematics, University of Missouri, Columbia, Missouri 65211

E-mail address: dale@cutkosky.math.missouri.edu 